\title{
9. Rhetorisierung und Rhythmisierung der Rede: Das Prosagedicht im Expressionismus
}

Allem Anschein nach folgt in der Moderne die Entwicklung neu entstehender Gattungen - zumindest bis zu ihrer völligen Einschmelzung in das jeweilige kulturelle Ensemble künstlerischer Gestaltungsoptionen - einem bestimmten Verlaufsmuster. Dieses ergibt sich aus den Funktionsregeln literarischer Kommunikation in einem ausdifferenzierten Sozialsystem Kunst. Dem Moment der Distinktion kommt dabei - wie gezeigt - oberste Priorität zu. Distinktionsgewinne nun lassen sich am leichtesten über Innovationen herstellen, weil die Kategorie der Neuheit eine ästhetische Basisdifferenz markiert zwischen dem bereits Existierenden und dem noch Unvertrauten. Für Kulturschaffende, die sich selbst im Sektor der seingeschränkten Produktion verorten, erscheint deshalb prinzipiell jede Form von Innovation interessant. Im Fall von Textsorten freilich kollidiert das strukturell geforderte Bestreben nach Erneuerung mit einer mehr oder weniger festgefügten symbolischen Ordnung akzeptierter Ausdrucksweisen, die Verstöße gegen ihre Grundlagen gemeinhin sanktioniert und insofern ein mächtiges Hindernis für die Einführung neuer Vertextungskonventionen darstellt. Die weitaus meisten Innovationen ereignen sich deshalb selbst in der Moderne im Rahmen bestehender Genremodelle. Am ehesten gelingt eine Erweiterung des Textsortenspektrums noch durch die Übernahme fremdkultureller Gattungskonzepte, weil hier ein willkommener Rechtfertigungsgrund für die Akzeptanz eines an sich prekären Innovationsaktes gegeben ist. Das Prosagedicht kann denn auch als Paradebeispiel für eine Genreneuheit gelten, die auf dem Weg rezeptiven Kulturtransfers Eingang in das deutsche Literatursystem findet.

Die erste Phase der Gattungsentwicklung zeichnet sich für gewöhnlich durch die vielfältigen, von begeisterter Zustimmung bis hin zu völliger Ablehnung reichenden Reaktionen aus, mit denen die Existenz des neuen Vertextungsmusters beantwortet wird. Da die Distinktionsgewinne bei der Nutzung der Novität für literarische Anfänger eindeutig größer sind als für bereits etablierte Schriftsteller, sorgen zumeist die Debütanten dafür, daß das Genre zu einer weithin akzeptierten Ausdrucksform avanciert. Mit jedem Zuwachs an Bekanntheit sinkt allerdings auch die Reputation der einstmaligen Innovation, weil damit zwangsläufig eine "Banalisierung des Entbanalisierungseffektes ' ' einhergeht, aus dem die Textsorte ursprünglich ihre Legitimation bezogen hat. So kommt es zu einer Aufspaltung der zuvor linear verlaufenden Evolution in zwei Entwicklungsstränge, die sowohl in symbolischer wie in ökono- 
mischer Hinsicht konträren Prämissen folgen. Ab diesem Punkt des geschichtlichen Verlaufs verändert sich der kommerzielle Gattungssektor kaum mehr, auch wenn es zuweilen zu merkwürdigen Interferenzbeziehungen zwischen diesem und seinem Widerpart kommen kann. Die Entwicklungsdynamik des Genres verlagert sich also weitgehend auf den experimentellen Sektor. Doch auch dieser stagniert vorübergehend, weil die eingetretenen Epigonalisierungstendenzen den Status der Textsorte schwächen und die Kulturproduzenten erst einen eigenständigen Funktionsbereich für die Novität finden müssen, der ihr eine Weiterexistenz auch dann erlaubt, wenn sich der von ihr ausgehende Erneuerungseffekt abgenutzt hat. Meist findet nun eine Ankoppelung an bereits bestehende Vertextungsmuster statt, die - vom Imagetransfer profitierend - dadurch ihrerseits einen Revitalisierungsschub erfahren und wieder dem Fundus künstlerischer Gestaltungsoptionen zugeführt werden. Sobald die Autoren die solcherart einsetzende Integration in das Gattungsensemble nutzen und die Tatsache der sektoriellen Spaltung als Gelegenheit begreifen, in Absetzung von den trivialen Mustern bislang nicht dagewesene Texthybriden zu entwickeln, erreicht die Genreentwicklung eine weitere Stufe. Der Zeitpunkt, zu dem dies geschieht, markiert den Beginn der dritten Phase im Verlauf der Evolution einer Textsorte unter den funktionalen Bedingungen der Moderne.

Im allgemeinen geht eine derartige Entdeckung neuer Profilierungschancen abermals von den Neuzugängern zum Literaturbetrieb aus, weil es sich für arrivierte Autoren nicht lohnt, möglicherweise unergiebige und deshalb riskante Bereiche des Gattungsspektrums auszuloten. Und so kann es auch kaum verwundern, wenn das Prosagedicht nach einer längeren Stagnationsphase im Anschluß an die Jahrhundertwende erst von einer jüngeren Generation von Schriftstellern wiederentdeckt und refunktionalisiert wurde. Bei dieser Gruppe handelt es sich um Vertreter jener Literaturbewegung, die unter dem Schlagwort IExpressionismus zu Bekanntheit gelangte. Obgleich die Poetik ihrer Protagonisten zum Teil überaus heterogen ist, lassen sich doch im Hinblick sowohl auf die Genrepräferenzen als auch auf bestimmte Muster der Handhabung von Sprache relativ weitreichende Gemeinsamkeiten erkennen, die es erlauben, die Indienstnahme des Prosagedichts im Expressionismus überblickshaft nachzuzeichnen, ohne jedem der behandelten Autoren ein eigenes Unterkapitel widmen zu müssen. Da sich die einzelnen Formen der Nutzung obendrein überwiegend als Reprisen bereits stattgehabter Funktionalisierungsakte erweisen, gelingt es einer synoptischen Darstellung zudem besser, übergreifende Strukturen der Gattungsentwicklung herauszuarbeiten.

Die expressionistische Dichtung insgesamt ist wohl am besten zu verstehen als ebenso heroischer wie verzweifelter Versuch, den aus der forcierten Sprachskepsis der Moderne resultierenden Aporien literarischer Kommunikation durch eine radi-

1 Pierre Bourdieu: Die Regeln der Kunst. Genese und Struktur des literarischen Feldes, S. 402 . 
kale Reformierung verbalen Ausdrucks zu entkommen. ${ }^{2}$ Zu diesem Zweck reinszeniert die um 1890 geborene Autorengeneration einen ähnlichen programmatischen Distanzierungsakt wie die ihr vorangehende Gruppe der Naturalisten. Während letztere sich vor allem von der epigonalen Gründerzeitlyrik abgrenzte, erklären die Expressionisten die Vertreter ästhetizistischer Formkunst zu ihren Hauptgegnern. So schreibt etwa Rudolf Kayser: "Wieder einmal gilt es [...], alte Formvorstellungen zu zertrümmem: diesmal die der l'art pour l'art-Epoche. ${ }^{3}$ Und Yvan Goll proklamiert: »Expressionismus [...] verleugnet jene Kunstgattungen des l'art pour l'art, denn er ist weniger eine Kunstform als eine Erlebnisform. ${ }^{\dagger}$ Die bei Goll zum Vorschein kommende, prononcierte Präferenz des sLebens` gegenüber der Kunst läßt im übrigen bereits erkennen, daß der Expressionismus nicht einfach als literarische Stilrichtung betrachtet werden darf, sondern daß er sich vielmehr als umfassende künstlerische Reformbewegung begreift, die darauf abzielt, Kunst in den Lebensvollzug zu integrieren und ihr so erneut gesellschaftliche Relevanz zu verschaffen.

Weil die Expressionisten nichts weniger als ein ästhetisches Programm sozialer Entdifferenzierung entwerfen, setzen ihre Innovationsbestrebungen allesamt bei der Sprache als sLeben und Kunst verbindendem Kommunikationsmittel an, deren Rolle als Gestaltungs- und deren Eignung als Verständigungsmedium systematisch reflektiert werden. Um den verbalen Ausdruck grundlegend zu erneuern, ohne in die klischierten Muster der Alltagsrede zurückzufallen oder zur Hermetik der l'art-pourl'art-Dichtung ${ }^{5}$ Zuflucht nehmen zu müssen, suchen die Autoren angestrengt nach

2 Vgl. hierzu Karl Pestalozzi: Sprachkritik und deutsche Literatur im 20. Jahrhundert. Rektoratsrede gehalten an der Jahresfeier der Universität Basel am 30. November 1990. Basel: Helbing und Lichtenhahn 1990 (= Basler Universitätsreden 86) und Dirk Göttsche: Die Produktivität der Sprachkrise in der modemen Prosa. Frankfurt a.M.: Athenäum 1987 (= Hochschulschriften Literaturwissenschaft 84).

3 Theorie des Expressionismus. Hrsg. von Otto F. Best. Stuttgart: Reclam 1976 (= Reclams Universal-Bibliothek 9817), S. 169. Ähnlich heißt es in Emst Stadlers Aufsatz Die nest franzäsische Lyrik (1912) mit bezeichnender Wendung gegen George: „Dieser Dichtung gegenüber, [...] die, wählerisch und exklusiv, immer mehr in toten Formeln erstarrt, muß heute in Deutschland jeder Versuch ermutigt werden, den einschnürenden Ring des Formalismus zu durchbrechen [...]. Für die deutsche Lyrik wenigstens liegt heute das Heil bei den scheinbar Formlosen. Wieder muß ein kostbares Gefäß, eine wundervolle, aber in ihren Nachbildungen bereits entwertete Form zerschlagen werden, damit für neue Erlebnisinhalte Platz werde. Emst Stadler: Dichtungen, Schriften, Briefe. Kritische Ausgabe, S. 427. Zur Ästhetizismuskritik der Expressionisten vgl. Helmut Gier: Die Entstehung des deutschen Expressionismus und die antisymbolistische Reaktion in Frankreich. Die literarische Entwicklung Emst Stadlers. München: Fink 1977 (= Münchner Germanistische Beiträge 21).

4 Yvan Goll: Die Lyrik in vier Bänden. Bd. 1: Frühe Gedichte 1906-1930. Hrsg. und kommentiert von Barbara Glauert-Hesse im Auftrag der Fondation Yvan et Claire Goll, SaintDié-des-Vosges. Berlin: Argon 1996, S. 9.

5 Vgl. Annette Simonis: Literarischer Ästhetizismus. Theorie der arabesken und hermetischen Kommunikation der Moderne. 
Artikulationsweisen, die von der Inflationierung der Sprache in der Moderne noch nicht entwertet worden sind. Goll gelangt in diesem Zusammenhang zu dem Schluß: "Zum Ausdruck neuen Empfindens gehörte eine Ursprache« (MuD-E, S. 614). Da eine solche freilich nicht einfach synthetisch erzeugt werden konnte, blieb zunächst nur der Rekurs auf vergessene oder außer Gebrauch geratene Redeformen. Oswald Pander formuliert in seinem Aufsatz Revolution der Sprache (1918) daher als Zielperspektive der jungen Generation: "Expressionismus will dem Erleben abgestorbene Sprache aus Erleben neu gebären« (MuD-E, S. 613). Goll konkretisiert diese noch recht allgemein gehaltene Forderung dann in wünschenswerter Deutlichkeit, wenn er am 25. Dezember 1921 an Zdenek V. Kalista schreibt: "Wir wollen wilde Ur-Gesängea ${ }^{6}$.

Archaische Ausdrucksmuster sollen also das Innovationspotential liefern, das für eine zeitgemäße Erneuerung der Dichtungssprache als nötig erachtet wird. Denn nur hier seien jene gestalterische Unnormiertheit und jenes wirkungsästhetische Potential zu finden, die nach Einschätzung der Expressionisten einen Ausweg aus der eingetretenen Krise künstlerischer Gestaltung weisen können: »Das Urgedicht [...] war pathetisch, weil es aus Leidenschaft entstanden war, pathetisch, weil es Leidenschaft erzeugen wollte [...], war eine Ansprache an die Menge, eine Mahnung, eine Anfeuerung, eine Ekstatik, eine direkte elektrische Entladung von Gefühl zu Gefühl. ${ }^{7}$ Die Elemente, die Stefan Zweig an den Frühformen der ’Poesies positiv hervorhebt, bezeichnen auch die Gestaltungsziele der expressionistischen Bewegung. Die Entwicklungsgeschichte der Literatur beschreibt er folgerichtig als langandauernden Degenerationsprozeß, in dem die Dichtkunst wimmer mehr lyrisch [...] und immer weniger pathetisch “ geworden sei: »Immer mehr entfernte sich das Gedicht von der Rede, immer mehr verlor es von jenem geheimnisvollen pathetischen Feuer, das nur genährt wird vom [...] Gegenüberstehen einer erregten Menge ${ }^{8}$.

Was die moderne Dichtung depotenziert habe, sei mithin die aus den Erfordernissen einer Schriftkultur resultierende Trennung der Produzenten und Rezipienten von Literatur, die letztlich dazu geführt habe, daß Lyrik monologisch geworden sei. Um nun diesen Zustand zu beenden, müsse die Dichtkunst wieder dialogisch angelegt und mit einem direkten Adressatenbezug versehen werden. Gestaltungstechnisch bedeutet dies, daß eine direkte Kommunikationssituation, wie sie beim münd-

6 Zitiert nach: Michael Knauf: Yvan Goll. Ein Intellektueller zwischen zwei Ländern und zwei Avantgarden. Bem/Berlin/Frankfurt a.M./New York/Paris/Wien: Lang 1996 (= Contacts II/19), S. 55, Anm. 62.

7 Expressionismus. Der Kampf um eine literarische Bewegung. Hrsg. von Paul Raabe. München: Deutscher Taschenbuch Verlag 1965 (= dtv sonderreihe 41), S. 15. Ähnliche argumentative Begründungsmuster begegnen schon in den poetologischen Schriften von Schlaf und Holz.

8 Ebd., S. 15f. Der Text Das newe Pathos (1909) wurde vier Jahre später dann als Einleitung der gleichnamigen expressionistischen Zeitschrift nachgedruckt. 
lichen Vortrag herrscht, simuliert und der literarische Ausdruck wirkungsästhetisch akzentuiert wird. Pathos sei dabei jener Sprachgestus, der das moderne Publikum am ehesten erreichen und es zur "Tat" anregen könne. Er entstehe durch eine gezielte Re-Rhetorisierung der $\curvearrowright$ Poesie, die freilich zugieich mit einer Verabschiedung traditionell lyrischer Formelemente wie Reim und Metrum einhergehen müsse: »Milde maßvolle Metrik« soll fortan durch wwilde maßlose Rhythmik (MuD-E, S. 619) ersetzt werden, wie etwa Herwarth Walden in seinem Essay Das Begriffiche in der Dichtung (1918) fordert. ${ }^{10}$

Genau besehen knüpfen die Expressionisten damit direkt an die zeitgenössische Debatte um den IRhythmusı in der Dichtung an. Auch für sie ist 2 Rhythmusı eine Art universales Lebensprinzip, nur deuten sie es unter dem Eindruck von Nietzsches Philosophie in affirmativer Emphase zugleich als zentrales Element des Dionysischen. ${ }^{11}$ Rhythmizität steht demnach für eine dynamische Seinsform, die sich den herkömmlichen Normen der Ästhetik entzieht. So deklariert Lothar Schreyer kurzerhand: "Der Rhythmus ist das Gestaltungsprinzip der Gegenwart. [...] Unvollkommen, unendlich ist der Rhythmus. Er ist die Auflösung jedes Maßes. Das Kunstwerk der Gegenwart ist aharmonisch, ist rhythmisch. ${ }^{12}$ Bezogen auf die Literatur bedeute dies: „Der Vers unserer Dichtung ist eine rhythmische Einheit, ohne Metrum, aharmonisch. ${ }^{13}$ Doch der aufs Totale gerichtete Anspruch des RhythmusBegriffs unterminiert letztlich auch die innerästhetischen Grenzziehungen. Gegenüber der unifizierenden Macht dieses Theorems verblaßt eine Einteilung in konkurrierende Ausdrucksmodi. Das Fazit lautet daher: "Es gibt keine Poesie und keine Prosa. Es gibt auch in der Sprache nur Kunst und Nichtkunst. ${ }^{14}$

$\mathrm{Da}$ die meisten expressionistischen Autoren dementsprechend Textformen mit hohem Rhetorisierungsgrad im Übergangsbereich von gebundener und ungebundener Rede bevorzugten, rückten mit einem Mal wieder die typischen Muster spoetischer Prosa in den Mittelpunkt des Interesses. Deshalb kann beispielsweise Ernst Stadler eine eigenwillige Langversdichtung wie Die geflügelte Erde. Ein Lied der Liebe und der Wunder um sieben Meere (1910) von Max Dauthendey zum Muster einer Form

9 Ebd., S. 18. Einige Jahre später dann warf Friedrich Gundolf in seinem George-Buch (1920) der neupathetischen Dichtung vor, sie habe durch ihren "Mangel an Grenze, Maß und Mitte« eine "Zerlösung der Formen« betrieben, die bis zur "Entstaltung" geht; ebd., S. 164.

In Lothar Schreyers Programmschrift Expressionistische Dichtung (1918/19) heißt es dann: "Die feste metrische Gestalt schafft keine Dichtung. Sie [...] schmückt eine Tatsache, sie macht die unpoetischer Tatsache spoetischı.» Theorie des Expressionismus. Hrsg. von Otto F. Best, S. 174.

11 Nietzsche hatte in der Götzen-Dämmerung bekanntlich ja den »Rausch» zur unabdingbaren "physiologischen Vorbedingung đer Kunst erklärt; Friedrich Nietzsche: Sämtliche Werke, Bd. 6, S. 116.

12 Theorie des Expressionismus. Hrsg. von Otto F. Best, S. 173.

13 Ebd., S. 175.

14 Ebd., S. 174. 
von Sprachverwendung erklären, die um kleinliche ästhetische Normen unbekümmert ist:

Die neue Haltung des Ich zur Welt, die im Formalen die losere Bindung, die Entspannung des metrischen Gefüges, die Lockerung der Rhythmik, die Aufgabe einer wählerischen und aristokratischen Diktion zur Folge hat, findet im heutigen Deutschland keinen gewichtigeren Verkünder als Max Dauthendey. Dauthendeys Form ist ganz gelöst, ganz weich, nachgiebig, flexibel und danum wie keine andere fähig, alle Bilder der Außenwelt ebensowohl wie die feinsten Schwingungen der Seele in sich zu sammeln. [...] Seine Langzeilen nähem sich einer rhythmisierten Prosa, zusammengehalten und abgeteilt nur durch die lockere und freischaltende Bindung der Reime. ${ }^{15}$

Das große moderne Vorbild für eine gelungene Fusionierung von Vers und Prosa im Stil erhabener Sprache gibt aber vor allem Nietzsche ab. ${ }^{16}$ Stadlers Aussagen im Manuskript einer Vorlesung über die Geschichte der deutschen Lyrik der neuesten Zeit (1914) entsprechen daher dem allgemeinen Gruppenkonsens der Expressionisten:

Nietzsche hat zunächst sprachlich gewirkt. Die inspiratorische Gewalt des im Zarathustra geschaffenen lyrischen Prosastils [...] hat im stärksten Maße auf die lyrische Sprache der nachfolgenden Zeit gewirkt. [...] Freilich konnte dieser Sprachstil erst wirken, nachdem der eigentliche Naturalismus überwunden war. Der Naturalismus hat sprachlich nichts bewirkt als eine Auflockerung des Materials und eine Beseitigung erstarter Conventionen. Noch bei Liliencron etwa ist nichts von der neuen dichter[ischen] Sprache zu spüren ${ }^{17}$.

Als zweite zentrale Bezugsfigur neben Nietzsche fungiert - was nicht weiter verwundern kann - Whitman, der beispielsweise von Max Picard regelrecht zum »Künder des Expressionismus« erklärt wird:

Der Expressionist liebt Whitman, weil durch den Anruf die Dinge aus dem Chaos heraus fixiert werden. Er ruft aber die gleichen Worte lauter als sie Walt Whitman gerufen, denn es ist für ihn eine größere Not, vom Chaos gehört zu werden als für Walt Whitman. Der Expressionismus ruft darum laut und ist pathetisch, nicht aus einem primären Lebensgefüh/ heraus, sondem aus Zwang. (MuD-E, S. 568) ${ }^{18}$

15 Emst Stadler: Dichtungen, Schriften, Briefe. Kritische Ausgabe, S. 329.

16 Würffel bemerkt denn auch zu Recht: "Der entscheidende Bezugspunkt der Verkündigungsdichtung der Moderne findet sich [...] in der wirkungsmächtigen Vermittlungsfunktion der poetischen Rede Nietzsches. Kein Werk hat das apologetische Sprechen in der Literatur auch nur annähernd so stark beeinflußt wie Nietzsches Also sprach Zarathustrox; Stefan Bodo Würffel: Verkündigung als literarische Form. In: Die Bibel in der deutschsprachigen Literatur des 20. Jahrhunderts. Hrsg. von Heinrich Schmidinger in Verbindung mit Gottfried Bachl, Johann Holzner, Karl-Josef Kuschel, Magda Motté und Walter Weiss. Redaktion: Dorit Wolf-Schwarz. Bd. 1: Formen und Motive. Mainz: Matthias-Grünewald-Verlag 1999, S. 207. Vgl. des weiteren Michael Landmann: Zum Stil des Zarathustra. In: M. L.: Geist und Leben. Varia Nictzscheana. Bonn: Bouvier 1951, S. 123-161, und Claudia Crawford: Nietzsche's Great Style: Educator of the Ear and of the Heart. In: Nietzsche-Studien 20 (1991), S. 210-237. 
Dadurch, daß beide Autoren in ihren Texten die "Bibelsprache« in einer bis dahin nicht dagewesenen Radikalität »von ihrem religiösen Ursprung gelöst ${ }^{19}$ und sie zu einem beliebig einsetzbaren Redemodus umgebildet haben, erscheinen sie als zeitgemäße Neubegründer jener archaisch-rhetorischen Ausdrucksform, die als imaginäre Bezugsgröße von den Expressionisten kulturtheoretisch konstruiert worden ist. Das konkrete historische Ur-Bild für dieses Phantasma einer unmittelbar in den Lebensvollzug eingebundenen Dichtung vor der ästhetischen Ausdifferenzierung in einzelne Funktionsbereiche und Gattungen (>Poesier bzw. Prosa; Epik, Dramatik und Lyrik) geben die biblischen Psalmen ab, die mit ihrer nichtprosodischen Vertextungsweise ein willkommenes Gegenmodell zur metrikfixierten abendländischen Dichtungstradition darstellen. Rezipiert wurden die Psalmen freilich nicht im hebräischen Original, sondern in Luthers prosanaher deutscher Übersetzung, die entscheidend dazu beitrug, daß Textsegmente zu Langzeilen zusammengefaßt wurden und so als eine intermediäre Form zwischen Vers und Prosa aufgefaßt werden konnten. ${ }^{20}$ Ursprünglich bildete lediglich der parallelismus membrorum, unterstützt durch "Wortwiederholungen", "Assonanzen und Alliterationen $"$ "1 das Hauptmerkmal der althebräischen Poesie. Luther nun formte daraus einen suggestiven Sprachstil, der zum Inbegriff religiöser Verkündigung werden sollte. ${ }^{22}$ Mit fortschreitender Säkularisierung emanzipierte er sich indes von seiner sakralen Funktion und trat allmählich in Konkurrenz zu den Mustern der antiken Dichtung. Nachdem der durch Luther vermittelte Redegestus der Psalmen im Verlauf des 18. Jahrhunderts zu einer der Hauptquellen der spoetischen Prosar geworden war und er sich um 1800 auch noch mit freirhythmischen Gestaltungstraditionen amalgamiert hatte, lagen bereits alle wesentlichen Voraussetzungen für eine ungehinderte ästhetische Verwendung dieses Ausdrucksregisters vor. Doch erst Whitman und Nietzsche schufen jene strahlkräftige Form erhaben-pathetischer Rede jenseits prosodischer Regelzwänge, die dann zu einem Leitkonzept der expressionistischen Generation werden sollte: Whitman übertrug die spätestens seit dem 18. Jahrhundert etablierte pantheistische Feier der Natur

allem der Aufsatz von A. Drey: Walt Whitman. In: Die Aktion 1 (1911), Heft 29, Sp. 907, sowie Johannes R. Bechers Nachschrift für Bruder Whitman in dem Band Das neue Gedicht (1918).

19 Inka Bach/Helmut Galle: Deutsche Psalmendichtung vom 16. bis zum 20. Jahrhundert. Untersuchungen zur Geschichte einer lyrischen Gattung. Berlin/New York: de Gruyter 1989 (= Quellen und Forschungen zur Sprach- und Kulturgeschichte der germanischen Völker N.F. 95), S. 313. Bach und Galle sprechen im Hinblick auf die luthersche Psalmenübersetzung von einem mschwebenden Übergang von Poesie in Prosarc; ebd., S. 321.

21 Ebd., S. 37. Doch bereits bei den hebräischen Psaimen liegt cine "prosanahe parataktische Versbindungu vor; ebd., S. 1.

22 Vgl. Martin Luther und seine Bedeutung für die deutsche Sprache und Literatur. Wissenschaftliche Konferenz Karpacz, 3-7.8.1983. Wroclaw: Wydawnictwo Universytetu Wroclawskiego 1985 (= Acta Universitatis Wratislaviensis 864; Germanica Wratislaviensia 64). 
auf die Errungenschaften moderner Zivilisation, und Nietzsche artikulierte seine radikale Metaphysikkritik blasphemischerweise gerade mit Hilfe biblischer Sprachschablonen. ${ }^{23}$ Die bei beiden erfolgte entdifferenzierende Verkoppelung ästhetischer und nichtästhetischer Diskurse (Philosophie, Historiographie, Naturwissenschaft) ${ }^{24}$ begünstigte die Verwendung des genus sublime/stylus grande als allgemeines dichterisches Rederegister zusätzlich. ${ }^{25}$

Allerdings hatte diese Universalisierung eines sprachlichen Gestus auf die Dauer gesehen einschneidende Konsequenzen für das Literaturverständnis der Expressionisten. So zog sie beispielsweise eine Reetablierung vermeintlich ausgelöschter ästhetischer Demarkationslinien nach sich. Um nämlich die Wirksamkeit hochgespannter Rhetorik gewährleisten zu können, mußte der dichterische Wortgebrauch von weniger inspirierten Weisen des Ausdrucks abgerückt werden. Und da die Art der Sprachgebung ja nicht an bestimmte Genres gebunden war, blieb nur eine dezisionistische Entscheidung auf Grund der Stilhöhe. Faktisch führte dies zu einer kategorischen Trennung zwischen Literatursprache einerseits und begrifflicher Alltagsrede andererseits. ${ }^{26}$ Unversehens wurde damit auch die vormals suspendierte Grenzziehung zwischen Lyrik und Prosa wiederhergestellt. So erklärt etwa Friedrich Kurt Benndorf in seinem Aufsatz Vom byrischen Idiom mit Bestimmtheit:

Obwohl die lyrische Dichtersprache ihre Ausdnucksmittel mit der Prosasprache teilt, ist sie doch von dieser beinahe ebensoweit entfernt, wie die Sprache der Töne, Farben oder Linien von der Wortsprache. Sie hat ihre eigene [...] Bildlichkeit (Metaphorik), ihre eigene Klanglichkeit (Melodik), ihre eigene Wortbewegung (Rhythmik) und ihr eigenes Tempo. Sie ist ein Idiom. ${ }^{27}$

Eine solcherart kategorisch behauptete Exklusivität der "Dichtersprache« aber deckt sich nicht nur mit ästhetizistischen Positionen, sie führt auch die angestrebte Verbindung von Kunst und Leben gänzlich ad absurdum. Und so bietet sich der Ex-

23 So behauptete Nietzsche in seinen nachgelassenen Fragmenten selbstbewußt: „Die Sprache Luthers und die poetische Form der Bibel als Grundlage einer neuen deutschen Poesie: das ist meine Erfindung." Friedrich Nietzsche: Sämtliche Werke, Bd. 11, S. 60. Siehe hierzu etwa Anne Tebartz-von Elst: Ästhetik der Metapher. Zum Streit zwischen Philosophie und Rhetorik bei Friedrich Nietzsche. Freiburg/München: Alber 1994, und Howard J. Waskow: Whitman: Explorations in form. Chicago/London: University of Chicago Press 1966.

$25 \mathrm{Daß}$ bei der Revitalisierung pathetischer Rede der hoher Ton und die erhabene Stillage bei Bedarf auch gezielt durch umgangssprachliche Elemente konterkariert und durch die Konfrontation mit iniedrigen Inhalten gebrochen werden konnten, versteht sich von selbst. Am konsequentesten wurde sie vom Sturm-Kreis propagiert: $» D i e$ Schriftstellerei erfült in der Sturm-Ästhetik die Funktion Verständigung/Verständlichmachung, die Dichtung die Funktion Kündung und Offenbarung; das Erfahrene oder zumindest Erfahrbare wird dem grundsätzlich Unbegreifbaren gegenübergestellt.«, Volker Pirsich: Der Sturm. Eine Monographie. Herzberg: Traugott Bautz 1985, S. 250.

27 Friedrich Kurt Benndorf: Vom lyrischen Idiom. In: Der Sturm 1 (1910/11), Heft 43, S. 341. 
pressionismus insgesamt als hochwidersprüchliches Literaturprogramm dar, das trotz einiger wegweisender Innovationen - und entgegen seinem erklärten Anspruch - in vielerlei Hinsicht stark traditionsverhaftet bleibt und bestehende Denkfiguren lediglich variiert.

Dies trifft nun auch und in besonderem Maße für den Umgang mit der Gattung Prosagedicht zu, die in der zweiten Dekade des 20. Jahrhunderts einerseits eine Konjunktur erfuhr, welche über das bisher Gekannte noch hinausging, die andererseits aber einer derartigen Abschleifung generischer Distinktionsmerkmale ausgesetzt war, daß sie fast bis zur Unkenntlichkeit entstellt wurde und längere Zeit nur noch in ihren epigonalen Formen überdauerte. Die expressionistische Tendenz zur Entdifferenzierung des Textsortenspektrums bewirkte in Verbindung mit der gleichzeitigen Auratisierung von 'Dichtungr im Endeffekt, daß die Kennzeichen spoetischer Rede mit einem Mal für alle Arten literarischen Ausdrucks verbindlich wurden. Trennte die Grenze zwischen sPoesier und Prosa bislang zwei ästhetische Areale, die - zumindest prinzipiell - beide auf künstlerische Dignität Anspruch erheben konnten, so wurde jetzt mit der "Prosasprache« ein im bisherigen Verlauf der Moderne immer wichtiger gewordener Bereich literarischer Gestaltung aus dem Bezirk der »Wortkunst« ausgegliedert. Die Expressionisten führten damit jene auf die Rückgewinnung ästhetischer Exklusivität zielende Ächtung der Prosa, ${ }^{28}$ die von den Protagonisten des l'art pour l'art propagiert worden war, ungeniert fort - mit dem einzigen, freilich gewichtigen Unterschied, daß sie nicht mehr, wie der Kreis um Stefan George, maass und reim "29 als verbindliche Poetizitätskriterien ansahen, sondern nichtprosodische Kunstmittel bevorzugten. ${ }^{30}$ Gleichwohl näherten die ausgiebige Verwendung von Pathosformeln, die fast durchgängige Metaphorisierung und die wirkungsästhetisch motivierte Re-Rhetorisierung der Sprache den literarischen Ausdruck auch hier traditionellen lyrischen Gestaltungsmustern soweit an, daß man im Hinblick auf das sog. expressionistische Jahrzehnt pauschalisierend vom "Zeitalter der Lyrik“" sprechen konnte. Ein weiteres Mal fungierte damit die „Poesie« als »Paradigma der literarischen Avantgarde« (MuD-E, S. 631). ${ }^{32}$

28 Fuchs charakterisiert die Prosa in diesem Zusammenhang pointiert als Redemodus mverschmutzer Autopoiesis«; Peter Fuchs: Moderne Kommunikation. Zur Theorie des operativen Displacements. Frankfurt a.M.: Suhrkamp 1993, S. 198. Blätter für die Kunst, 2. Folge, Bd. 2, März 1894, S. 34.

30 Doch selbst unter den expressionistischen Autoren gab es Schriftsteller, die sich weiterhin überwiegend an den Normen der Prosodie orientierten. Vertreter dieser Fraktion sind vor allem Georg Heym und Jakob van Hoddis [= Hans Davidsohn]. Gleichwohl hatte Heym zwischenzeitlich vor, "Gedichte in Prosan kombiniert mit "Novellena zu publizieren, wie aus einem Brief an Emst Rowohlt vom 1. Juli 1911 hervorgeht; Georg Heym: Dichtungen und Schriften. Gesamtausgabe, hrsg. von Karl Ludwig Schneider. Bd. 3. Hamburg/München: Ellermann 1960, S. 255f.

31 Peter Scher [= Fritz Schweynert]: Das Zeitalter der Lyrik. In: Die Aktion 3 (1912/13), Nr. 27, Sp. 645. 
Immerhin verschaffte die Präferenz freirhythmischer, nichtprosodischer Formen der Lyrik allen Spielarten der spoetischen Prosar neue Akzeptanz, ja eine teilweise entlyrisierte Versdichtung verschmolz mit einer rhetorisch aufgeladenen, sprachlich reich ornamentierten Prosa zu einem in dieser Weise noch ungekannten intermediären Ausdrucksmodus, der als eine Art coincidentia oppositorum auf textueller Ebene und damit als moderne Verwirklichung der romantischen Ganzheitsutopie einer "Universalpoesie« präsentiert werden konnte. Als Gattungsnamen für diese Art von Versprosa mit dezidiert poetischem Anspruch fungierten oftmals Begriffe wie Gesang، oder `Dichtung ${ }^{33}$ die in ihrer unspezifischen Allgemeinheit auf ebenso sprimitives wie fundamentale Vertextungsweisen vor der Ausdifferenzierung in einzelne Genres verweisen. Wie die Konjunktur der Termini ,Dithyrambe//Dithyrambus ${ }^{34}$ und $/$ Hymne//Hymnus ${ }^{35}$ zeigt, kam es in diesem Zusammenhang auch zu einer problemlosen, synkretistischen Einbeziehung antiker Literaturgattungen. ${ }^{36}$ In vielen Fällen griffen die Autoren bei der Benennung ihrer Texte sogar zu Bezeichnungen, die aus dem religiös-liturgischen Kontext stammen: ${ }^{37}$, Gebet ${ }^{38}$, ,Psalm ${ }^{39}$, ,Requi-

32 Vgl. hierzu auch: Immanente Ästhetik - Ästhetische Reflexion. Lyrik als Paradigma der Modeme. Hrsg. von Wolfgang Iser. München: Fink 1966 (= Poetik und Hermeneutik 2).

Siehe etwa die Gedichtsammlungen Gesänge gegen den Tad (1914) von Gottfried Kölwel, Gesänge aus den drei Reichen (1917) von Franz Werfel und Versähnung. Gesänge und Psalmen (1919) von Oskar Schürer, Yvan Golls Buch Astral Ein Gesang (1920), den Gesang eines Verdammten an die seligen Geprïften der Erde und den Gesang einer Frax in Franz Werfels Band Der Gerichtstag (1919) oder die Texte Abgesang und Todesgesang in Johannes Urzidils Sturz der Vendammten (1919).

34 Die Wiederbelebung des Begriffs ,Dithyrambust ist natürlich Nietzsche zu verdanken, der - wie seine Schrift Die Geburt der Tragödie aus dem Geiste der Musik (1872) erkennen läßt völlig davon überzeugt war, daß der Mensch nur wim dionysischen Dithyrambus [...] zur höchsten Steigerung aller seiner symbolischen Fähigkeiten gereizt « werde; Friedrich Nietzsche: Sämtliche Werke, Bd. 1, S. 33. Allein von Yvan Goll existieren drei „Gedichtw-Bände, die diese Bezeichnung im Ober- oder Untertitel tragen: Ditbynamben (1918), Der newe Orphews. Eine Ditbyrambe (1918) und Der Torso. Stanzen und Ditbyramben (1918).

35 Siehe beispielsweise die Bände Hymnen (1913) von Ottokar Březina, Seplemberschrei. Hymnen, Gebete, Lästerungen (1920) von Ludwig Meidner oder die Sammlungen Verklärung. Hymne (1922) und Hymnen (1924) von Johannes R. Becher. Vgl. Hellmut Thomke: Hymnische Dichtung im Expressionismus. Bem: Francke 1972.

Nach Knauf wist die Tendenz zum Eklektizismus als Charakteristikum der Avantgardebewegungen insgesamt anzusehenc; Michael Knauf: Yvan Goll. Ein Intellektueller zwischen zwei Ländern und zwei Avantgarden, S. 123.

37 Wie Anz und Stark zu Recht bemerken, wird im Expressionismus »die Lyrik zum Medium des Metaphysikverlusts und gleichzeitig zum Ausdruck der ersatzmetaphysischen Erlösungsvisionen (MuD-E, S. 632). Schon Paul Zech zeigt sich in seinem Aufsatz Die Grundbedingung der modermen Lyrik (1913) davon überzeugt, daß sich win der modemen Lyrik tiefste Religiosität mit dem Wissen um den Verlust der Religion« vereinigen würden: „Was sie [= die Lyrik] in früheren Zeiten von der Religion empfing, das sucht sie in der modernen Zeit, der Zeit ihrer eigentlichen, selbständigen Entwickelung, der Religion wieder zuzuführen, an religiösen Inhalten selbst zu erschaffen.« (MuD-E, S. 642) Vgl. in diesem Zusam- 
em ${ }^{40}$, Kantate $^{41}$. Der damit gegebene Verweis auf den weiten Komplex kultischer Handlungen ist abermals klarer Beleg für das Bestreben, Redemuster mit sozialer Verbindlichkeit und tendenziell performativem Charakter zu schaffen. Auch ermöglicht die Formelhaftigkeit der Genres, auf die hier Bezug genommen wird, einen Gestus des literarischen Sprechens, der nicht auf das einzelne Individuum beschränkt ist, sondern sich als Teil kollektiver Rede versteht.

Wenn rhythmisierte Prosa in Buchform veröffentlicht wurde, geschah dies stets in Kombination mit Verslyrik; eigenständige Textbände mit Kurzprosa bewegen sich durchweg außerhalb des Radius des Gattungsmodells Gedicht in Prosa. ${ }^{42}$ Doch anders als bei Liliencron oder auch Bierbaum geht es in den Textsammlungen expressionistischer Autoren natürlich nicht darum, 'Poesier und Prosa miteinander zu kontrastieren. Vielmehr loten sie, ähnlich wie Dauthendey oder Schlaf, das gesamte Verlaufsspektrum dichterischer Gestaltungsweisen aus, wobei das Prosagedicht als selbstverständliche sprachliche Präsentationsform der Lyrik - oder vielleicht besser: eines die herkömmlichen Grenzen der Ausdrucksmodi übergreifenden Typs von Dichtkunst - erscheint. ${ }^{43}$ Als Beispiele für solche Gedichts-Publikationen, die Texte mit z.T. extremen, von Abschnitten rhythmischer Prosa nicht mehr unterscheidbaren Langzeilen enthalten, wären u.a. zu nennen: Georg Trakls Gedichte (1913), Ottokar Březinas Hymnen (1913), Ludwig Rubiners Das bimmlische Licht (1916), Yvan Golls Bände Requiem. Für die Gefallenen von Europa (1917), Der newe Orphess. Eine Ditby-

menhang auch Frank Krause: Sakralisierung unerlöster Subjektivität. Zur Problemgeschichte des zivilisations- und kulturkritischen Expressionismus. Frankfurt a.M./Berlin/Bern/ Wien: Lang 2000 (= Bochumer Schriften zur deutschen Literatur 57).

Siehe etwa die Texte Gebet für die Feinde in Ottokar Březinas Hymnen (1913), Gebel um Reinbeit und Gebet gegen Worte in Franz Werfels Gesänge aus den drei Reichen (1917), Gebet in Albert Ehrensteins Die rote Zeit (1917) oder die Stoßgebete in Theodor Taggers - besser bekannt unter dem Pseudonym Ferdinand Bruckner - Der zerstörte Tasso (1918).

Siehe u.a. Georg Trakls Psalm in seiner Textsammlung Gedichse (1913) und Johannes Urzidils Unteraxtlicher Psalm in dem Band Sturz der Verdammten (1919).

Siehe beispielsweise die Bände Requiem für die Gefallenen von Europa (1917) von Yvan Goll und Requiem (1921) von Georg Kulka.

Siehe die Mitternacbtskantate an alle Verlassenen in Johannes Urzidils Sturz der Verdammten (1919).

Vgl. etwa den Band Kobolz. Grotesken (1917) von Hans Reimann.

43 Obwohl vereinzelt auch Vers- in Prosatexte umgearbeitet wurden und umgekehrt, sind solche Transformationsakte insgesamt gesehen eher die Ausnahme. Beispiele für solche Umschriften sind die handschriftlichen Entwürfe und Drucke der beiden Texte Die Heffer sind tot und Stimme über Barbaropa von Albert Ehrenstein und die einzelnen Fassungen von Yvan Golls Der Panamakanal; vgl. Albert Ehrenstein: Werke. Hrsg. von Hanni Mittelmann. Bd. 4/II. O.O. [München]: Klaus Boer 1997, S. 196f. und 200f., sowie Joachim Müller: Yvan Goll im deutschen Expressionismus. Berlin (Ost): Akademie 1962 (= Sitzungsberichte der Sächsischen Akademie der Wissenschaften zu Leipzig. Philologisch-historische Klasse $107 / 2)$, S. 52-65. 
rambe (1918), Der Torso. Stanzen und Ditbyramben (1918), Ditbyramben (1918) und Die Unterwelt (1919), Mechtild Lichnowskys Gott betet (1918), Theodor Taggers Der zerstörte Tasso (1918), Franz Werfels Veröffentlichungen Gesänge aus den drei Reichen (1917) und Der Gerichtstag (1919), Johannes Urzidils Sturz der Verdammten (1919), Johannes R. Bechers Sammlungen An Alle. Neue Gedichte (1919), Evig im Aufrubr (1920), Um Gott (1921) und Verklärung. Hymne (1922) sowie Albert Ehrensteins Bücher Die rote Zeit (1917), Den ermordeten Brüdern (1919), Die Gedicbte (1900-1919) (1920) und Mein Lied (1932). ${ }^{44}$

Innerhalb der genannten Bände sind Einzeltexte nicht selten zu größeren Ensembles zusammengeschlossen, d.h. Verfahren der Zyklusbildung, wie sie aus dem Kontext der Lyrik vertraut sind, werden im Expressionismus auch auf Intermediärformen zwischen Vers und Prosa wie auch auf rhetorisierte Kurzprosa insgesamt angewandt. Diese Vorgehensweise führt im Ergebnis dazu, daß die konstitutive Differenz zwischen kurzen und langen literarischen Genres eine nachhaltige Aufweichung erfährt: Unspezifische Texttypen wie sDichtung oder 'Gesang sind eben umfangsungebundene Ausdrucksmuster, die sich im einen Fall nur über wenige Zeilen, im anderen aber auch über viele Seiten erstrecken können. Zuweilen füllen derartige Textgebilde eine ganze Buchveröffentlichung, wie etwa im Falle von Yvan Golls Bänden Requiem. Für die Gefallenen von Europa (1917) und Astral. Ein Gesang (1920). Auf diese Weise entstehen - je nach Vertextungsart - regelrechte Langgedichte, eposartige Versprosahybriden oder aus einzelnen Kurzprosatexten bestehende zyklische Formen. ${ }^{45}$

Derartige Transgressionsphänomene allerdings berauben das Prosagedicht vollends seiner ohnehin schwach ausgeprägten Gattungsspezifik. So kombiniert Albert Ehrenstein in Briefe an Gott (1922) 40 Kurzprosatexte mit 21 Versgedichten. Der

44 Viele der hier versammelten Langzeilentexte werden gewöhnlich umstandslos der Lyrik zugerechnet - eine Praxis, die den utopischen Charakter der ihnen zugrunde liegenden Ästhetik unterschlägt. Faktisch aber läßt sich - wie ein Blick auf die Texte zeigt - keine zuverlässige Abgrenzung vornehmen zwischen lyrischen und Prosa-Langzeilen, vielmehr existiert zwischen beiden ein weiter Übergangsbereich. Berücksichtigung fanden in der obrigen Aufzählung deshalb grundsätzlich alle Sprachgebilde mit signifikanten Textsegmenten, deren Umfang eine Druckzeile überschreitet. Nicht einbezogen wurden dagegen Bände, deren Langzeilentexte Endreime aufweisen, wie z.B. Ernst Stadlers Der Aufbruch (1914) oder Johannes R. Bechers Der Gestorbene (1922).

45 Siehe hierzu etwa Cordula Gerhard: Das Erbe der "Großen Form». Untersuchungen zur Zyklus-Bildung in der expressionistischen Lyrik. Frankfurt a.M./Bem/New York: Lang 1986 (= Europäische Hochschulschriften I/910), Wolfgang Braungart: Zur Poetik literarischer Zyklen. Mit Anmerkungen zur Lyrik Georg Trakls. In: Zyklische Kompositionsformen in Georg Trakls Dichtung. Szegeder Symposion. Hrsg. von Károly Csúri. Tübingen: Niemeyer 1996, S. 1-27, Robert Oeste: Arno Holz: The Long Poem and the Tradition of Poetic Experiment, und Thomas Rietzschel: »Prosa wird wieder Dichtungw. Die lyrische Tendenz expressionistischen Erzählens. In: Weimarer Beiträge 25 (1979), Heft 3, S. 75-89.

46 Vgl. Margherita Versari: Albert Ehrenstein: zwischen Religion und Postreligion. Die Briefe 
Umstand freilich, daß die einzelnen Texte keine Titel tragen und nur durch großgedruckte Initialbuchstaben als eigenständige Einheiten gekennzeichnet sind, hält das Verhältnis von Teil und Ganzem in der Schwebe. Zusätzlich infrage gestellt wird es dadurch, daß Ehrenstein kurz vor der Veröffentlichung der Briefe an Gott zwei Bände herausbrachte - Dem ewigen Olymp (1919) und Die Nacht wird (1921) -, die im Untertitel als »Erzählungen und Gedichte» bzw. »Gedichte und Erzählungen« ausgewiesen sind und damit ein Modell der publikatorischen Verknüpfung von Lyrik und Narrativik präsentieren, das mit der Textform Prosagedicht nichts zu tun hat. Noch unsicherer wird eine Genrezuordnung bei Mechtild Lichnowskys Gott betet benanntem Zyklus von acht mit römischen Zahlen durchnumerierten rhythmisierten Kurzprosastücken; da hier para- und kontextuelle Gattungssignale fehlen, können die Texte nur ais expressionismustypische Variationen des Grundmodells rhetorisierter Kurzprosa, nicht aber als Prosagedichte angesehen werden. Und wenn dann Prosasegmente ganz ohne Titel oder Ziffer bleiben und obendrein noch durch einen losen Handlungsfaden miteinander verbunden sind, wie bei Klabunds Der Neger (1920), sind schließlich auch die Grenzen in Richtung lyrisierter narrativer Großform überschritten.

Die im Expressionismus erfolgte durchgreifende Rhetorisierung der Versdichtung und die daraus resultierende partielle Annäherung an den Sprachmodus der sungebundenen Rede $^{47}$ universalisiert zwar das Modell einer "Prosalyrik«, wie es einst Halbe umrißhaft entworfen hat, entzieht aber gleichzeitig dem Prosagedicht als distinktem Texttyp die Voraussetzungen seiner Existenz. Damit erlebt das Genre innerhalb des deutschen Sprachraums die zweite fundamentale Krise in seiner Geschichte, die sich im Endeffekt als noch einschneidender erweist als die zuvor erfolgte Spaltung in zwei Zirkulationssphären. War die Gattung durch das Aufkommen und Überhandnehmen epigonaler Autoren zwar einer weitgehenden Banalisierung ihrer Vertextungsstrategien ausgesetzt, so gelang ihr durch die sich damit vollziehende Ausdifferenzierung der Leserschichten doch auch wieder eine Konsolidierung innerhalb eines eingeschränkten Distributionssektors. Die ungerichtete Erweiterung eines literarischen Verfahrens über alle Genreschranken hinaus aber beraubte das Prosagedicht nachhaltig seiner generischen Funktionslogik, so daß es nun tatsächlich mit dem textsorten- und diskursformationenübergreifenden Modus spoeti-

an Gott. In: Hans Schumacher (Hrsg.): Spiegel im dunklen Wort. Analysen zur Prosa des frühen 20. Jahrhunderts. Bd. 2. Frankfurt a.M./Bern: Lang 1986 (= Berliner Beiträge zur neueren deutschen Literaturgeschichte 11), S. 249-259.

47 Verschiedentlich werden auch wieder typographische Experimente durchgeführt. So kombiniert etwa Ludwig Rubiner in Das bimmlische Licht (1916) - ähnlich wie weiland CroissantRust - Textabschnitte, deren Anfangszeile eingerückt ist, mit solchen, die shängend gesetzt sind, d.h. die ab der zweiten Zeile einen Einzug aufweisen. Während erstere Praxis gewöhnlich auf Prosa verweist, läßt sich letztere meist bei Langzeilenlyrik wiederfinden. Rubiner mischt also die Ausdrucksmodi schon auf der Ebene der Typographie. 
scher Prosar verschmolz, den weite Teile der Forschung seit jeher als identisch mit dem poème en prose angesehen haben. ${ }^{48}$

An dieser Entwicklung konnte auch die verstärkte Rezeption der internationalen Gattungsenwicklung nichts ändern, die sich bei den expressionistischen Autoren beobachten läßt, zumal diese gleichfalls in erster Linie auf Sprachstrukturen gerichtet ist. Die im Anschluß an das Erscheinen von Karl Klammers Auswahlübertragung (1907) einsetzende Rimbaud-Begeisterung in Deutschland ${ }^{49}$ jedenfalls machte sich vor allem an der ungewohnten Bildlichkeit der Texte ${ }^{50}$ und ihren syntaktischen Kühnheiten fest, denn hier schien bereits jenes mraisonné dérèglement de tous les sens ${ }^{51}$ seine verbale Umsetzung gefunden zu haben, das auch die Expressionisten zu ihrer Zielperspektive erhoben. ${ }^{52}$ Der Umstand, daß Klammer unter dem Ge-

48 Vgl. Kapitel I/2. Weil die Vertreter dieses Forschungsstrangs die Gattung vorschnell mit einem bestimmten, historisch genau beschreibbaren Stadium ihrer Entwicklung gleichsetzen, gelangen sie zu falschen Rückschlüssen über Genese und Ausprägungsform des Genres.

Gsteiger konstatiert zu Recht: »Die deutsche Übersetzungsgeschichte Rimbauds und die Geschichte seiner Wirkungen auf die deutsche Literatur wird bis zum Ersten Weltkrieg und darüber hinaus beherrscht von Karl Klammer (K.L. Ammer)«; Manfred Gsteiger: Französische Symbolisten in der deutschen Literatur der Jahrhundertwende (1869-1914), S. 214. Wie sehr die Rezeption der Illuminations in Deutschland von der Generation expressionistischer Autoren getragen wurde, verdeutlicht u.a. die Tatsache, daß Rimbauds Prosagedichte noch wenige Jahre zuvor völlig unbeachtet blieben: „Das Ausmaß des Befremdens, das die Illuminations um 1900 ausgelöst haben [...], dokumentiert sich vorwiegend auf negative Weise, durch die Beharrlichkeit, mit der man diese Dichtungen in den in verschiedenen Zeitschriften erschienenen Rimbaud-Aufsätzen nicht zur Kenntnis nimmt. "Colette Faber-Bellion: Die Rimbaud-Übersetzung von K.L. Ammer. Beispiele aus den Illuminations. In: Studien zur Literatur des 19. und 20. Jahrhunderts in Österreich. Festschrift für Alfred Doppler zum 60. Geburtstag. Hrsg. von Johann Holzner, Michael Klein und Wolfgang Wiesmüller. Innsbruck: Institut für Germanistik 1981 (= Innsbrucker Beiträge zur Kulturwissenschaft. Germanistische Reihe 12), S. 137, Anm. 4.

50 Friedrich hat dafür bekanntlich den Terminus der mabsoluten Metapher geprägt, den er anhand von Rimbauds Le Bateau ivre erläutert; vgl. Hugo Friedrich: Die Struktur der modemen Lyrik, S. 55.

51 Arthur Rimbaud an Paul Demeny, 15.5.1871; Arthur Rimbaud: Euvres complètes. Édition établie, présentée et annotée par Antoine Adam. Paris: Gallimard 1972 (= Bibliothèque de la Pléiade 68), S. 251.

52 Rimbauds Vertextungspraxis traf sich vor allem mit den Forderungen des Sturm-Kreises nach einer antimimetischen und alogisch-unverständlichen, von der Alltagssprache weit abrückenden "Wortkunst" jenseits metrischer Normierung. Zur Ästhetik des Stum-Kreises vgl. Richard Brinkmann: Zur Wortkunst des Sturm-Kreises. Anmerkungen über Möglichkeiten und Grenzen abstrakter Dichtung. In: Unterscheidung und Bewahrung. Festschrift für Hermann Kunisch zum 60. Geburtstag, 27. Oktober 1961. Hrsg. von Klaus Lazarowicz und Wolfgang Kron. Berlin: de Gruyter 1961, S. 63-78; Elemir Terray: Zur Kunstauffassung des Berliner Sturm-Kreises. In: Philologica 18 (1966), S. 71-82; Wilderich Voermanek: Untersuchungen zur Kunsttheorie des Sturm-Kreises. Diss. (Masch.) FU Berlin 1970; Kurt 
samttitel mDichtungen» ein buntes Gattungspotpourri von 25 Versgedichten, 14 Prosagedichten aus den Illuminations (Erleuchtungen) und den thematisch miteinander verknüpften Kurzprosatexten von Une saison en enfer (Ein Sommer in der Hölle) versammelte, präsentierte Rimbaud obendrein als Autor, der sich um Genrekonventionen wenig oder gar nicht kümmert, und leistete dem Mißverständnis Vorschub, auch er würde einen textsortenübergreifenden Ausdrucksmodus zwischen Lyrik und Prosa anstreben. ${ }^{53}$ Dem deutschen Gattungsmodell Prosagedicht jedenfalls vermochte die Rezeption der Illuminotions kaum neue Impulse zu geben, ${ }^{54}$ was übrigens ebenso auf die mittlerweile relativ breite Präsenz der Petits poèmes en prose zutrifft, ${ }^{55}$ die im Ex-

Möser: Literatur und die »Große Abstraktion«. Kunsttheorie, Poetik und mabstrakte Dichtung im Sturm 1910-1930. Erlangen: Palm \& Enke 1983 (= Erlanger Studien 46); Malcolm S. Jones: Der Sturm: A Focus of Expressionism. Columbia (South Carolina): Camden House 1984 (= Studies in German Literature, Linguistics, and Culture 16); Volker Pirsich: Der Sturm, sowie Sven Anold: Das Spektrum des literarischen Expressionismus in den Zeitschriften Der Sturm und Die Weissen Bläter. Frankfurt a.M./Berlin/Bern/New York/Paris/ Wien: Lang 1998 (= Forschungen zur Literatur- und Kulturgeschichte 64).

Ein Blick auf die Illuminations scheint diesen Eindruck zunächst zu bestätigen, finden sich doch darin mehrfach Texte, die eingeführte Gattungsbezeichnungen als Titel tragen: Sonnet (Sonett), Conte (Märchen) oder auch Scines (Szenen). Auch enthält der Band neben den Kurzprosatexten das Versgedicht Marine (Seestück) sowie eine Reihe von Mischformen; vgl. hierzu Antoine Fongaro: Les Vers mesurés dans Illuminations. In: Littératures, Nr. 8, Herbst 1983, S. 63-79, A. F.: Segments metriques dans la prose d'Illuminations. Toulouse: Presses Universitaires du Mirail-Toulouse 1993 (= Les Cahiers de Littératures) sowie JeanPierre Bobillot: Rimbaud et le wvers librer. In: Poétique, Nr. 66, April 1986, S. 199-216. Derartige textuelle Störsignale zielen bei Rimbaud freilich darauf, das Funktionieren von „Poetizitäte an sich infragezustellen, und dienen nicht einer pathetischen Ausdruckssteigerung eines nicht ohne Naivität postulierten und letztlich auf Entdifferenzierung angelegten Gestaltungsmodus des ,Dichterischen;; vgl. Rolf Kloepfer/Ursula Oomen: Sprachliche Konstituenten modemer Dichtung. Entwurf einer deskriptiven Poetik. Rimbaud. Bad Homburg v.d.H.: Athenäum 1970, Tzvetan Todorov: Les Genres du discours. Paris: Éditions du Seuil 1978 (= Collection poétique) (Kapitel »Une complication de texte: les Illuminations und Michel Murat: Du vers à la prose: le dégagement de l'écriture. In: [Università degli studi di Pisa. Istituto di Lingua e Letteratura francese (ed.):] Atti del Colloquio Internazionale "Arthur Rimbaud: poesia e avventuran (Grosseto, 11-14 settembre 1985), a cura de Mario Matucci. Pisa: Pacini 1987, S. 59-68.

Das schließt natürlich nicht aus, daß die Wirkung in bestimmten Werkphasen einzelner Autoren u.U. beträchtlich war. So sind etwa Trakls Prosagedichte eindeutig wunter dem Einfluß Rimbauds entstanden'; Ludwig Dietz: Die lyrische Form Georg Trakls. Salzburg. Otto Müller 1959 (= Trakl-Studien 5), S. 191. Zu dessen Rimbaud-Rezeption allgemein vgl. Reinhold Grimm: Georg Trakls Verhälnis zu Rimbaud. In memoriam Karl Klammer, †8.3. 1959. In: Germanisch-romanische Monatsschrift N.F. 9 (1959), S. 288-315.

55 Nach der von Camill Hoffmann und Stefan Zweig besorgten Edition der Gedictste in Vers und Prosa (1902), deren Prosateil 1915 in der populären »Insel-Bücherei« noch einmal separat publiziert wurde, erschienen in Band 1 der ersten deutschen Werkausgabe von Baudelaires Schriften die von Margarete Bruns übersetzten Novellen und kleinen Dichtungen in Prosa (1904), ein von Fritz Gundlach zusammengestellter Band Gedichte und Skizizen (1908) im 
pressionismus besonders wegen ihres nüchtern sprosaischen Charakters wenig Resonanz fanden. ${ }^{56}$ Überhaupt war das Interesse an Baudelaire im zweiten Jahrzehnt des 20. Jahrhunderts im großen und ganzen ein eher historisches, weil die Wahrnehmung dieses Autors gerade in Deutschland überaus stark mit dem ästhetischen Konzept $\$ Symbolismus` verbunden blieb. ${ }^{57}$ Obgleich also die Expressionisten die erste Generation deutschsprachiger Autoren darstellen, denen mit den Prosagedichten Baudelaires und Rimbauds zwei der grundlegenden französischen Muster des Gattungsmodells in vollständigen Übersetzungen zugänglich waren, hatte die verspätetete Wahrnehmung der internationalen Ausprägungsformen keinen nennenswerten Einfluß mehr auf die eigenkulturelle Genreevolution.

Überhaupt gelangte die Entwicklung der Textsorte mit dem Expressionismus an einen vorläufigen Endpunkt. Die Futurismus-Rezeption des Sturm-Kreises öffnete die moderne Ästhetik für Experimente mit der Materialität des Sprachzeichens, welche herkömmliche textuelle Unterscheidungskriterien zunehmend obsolet erscheinen ließen. ${ }^{58}$ Denn ein semiotischer Umgang mit den Basiselementen der Schrift

Rahmen von Reclams »Universal-Bibliothekı sowie eine Gesamt- (1920) und eine Auswahlausgabe (1923) der Kleinen Gedicbte in Prosa.

56 Das Bild Baudelaires in Deutschland wurde nach wie vor von den Fleurs du mal geprägt, einem Werk also, dessen Texte sich besonders durch die "Strenge» ihrer "Rhythmik und der metrischen Gefüge» auszeichnen; Emst Stadler: Dichtungen, Schriften, Briefe. Kritische Ausgabe, S. 328.

57 Vgl. in diesem Zusammenhang Thomas Keck: Der deutsche "Baudelairew. Bd. 1: Studien zur übersetzerischen Rezeption der Fleurs du Mal. Bd. 2: Materialien. [1. Halbband: Bibliographie deutscher Übersetzungen Baudelairescher Vers-Lyrik; 2. Halbband: Arbeitsanthologie.] Heidelberg. Winter 1991 (= Beiträge zur neueren deutschen Literaturgeschichte. 3. Folge 111/112). Dem entspricht, daß das Prosagedicht konsequenterweise als Versuch einer Musikalisierung der Prosa wahrgenommen wird. So schreibt etwa Pick - die Widmungsvorrede an Arsène Houssaye naiv paraphrasiezend - über Baudelaire: „Gleich Flaubert träumte er das Wunder einer poetischen Prosa, die musikalisch wäre ohne Reim und Rhythmus.، Und er erreichte sein Ziel in den wunderbaren sDichnungen in Prosaur, Otto Pick: Baudelaire. In: Die Aktion 2 (1912), Sp. 557.

Vgl. Hansgeorg Schmidt-Bergmann: Die Anfänge der literarischen Avantgarde in Deutschland. Über Anverwandlung und Abwehr des italienischen Futurismus. Ein literarhistorischer Beitrag zum expressionistischen Jahrzehnt. Stuttgart: M \& P Verlag für Wissenschaft und Forschung 1991 (= M \& P Schriftenreihe für Wissenschaft und Forschung). White spricht im Hinblick auf Marinetti vom ikonischent Charakter futuristischer Texte; vgl. John J. White: The Argument for a Semiotic Approach to Shaped Writing. The Case of Italian Futurist Typography. In: Visible Language 10 (1976), S. 53-86. Chick hat die einzelnen Facetten dieses Ausdruckstyps summarisch benannt: "Marinetti experimented with the colour of the ink, the typeface, the size of the letters, the vertical and horizontal spacing, the left margin, and the spelling (repeating some letters several times for emphasis). J Jean $M$. Chick: Form as Expression. A Study of the Lyric Poetry Written Between 1910 and 1915 by Lasker-Schüler, Stramm, Stadler, Benn, and Heym. New York/Bem/Frankfurt a.M./ Paris: Lang 1988 (= Studies in Modem German Literature 10), S. 18. 
suspendiert die immer noch auf der Ebene der Semantik operierenden Codierungsformen von Poetizität bzw. redefiniert sie auf anderem Niveau. ${ }^{59}$ Die nachgerade programmatische Hinwendung zur visuellen Poesie ${ }^{60}$ und zum Lautgedicht ${ }^{61}$ wurde dann für den Dadaismus und den Surrealismus ${ }^{62}$ verpflichtend. Die Avantgardebewegungen des frühen 20. Jahrhunderts überboten so alle bisherigen Versuche zur Gattungsemeuerung systematisch in ihrer Radikalität und stellten sie verfahrenstechnisch in den Schatten. ${ }^{63}$ Auf Grund der historischen Relativierung seines transgressiven Potentials funktionierte nun auch die "Bedürfnisbefriedigung «, die das Prosagedicht ursprünglich im Ensemble der Genres geleistet hatte, nicht mehr in gleicher Weise, und der bislang schärfste textuelle Subversionsakt gegen die etablierten innerliterarischen Grenzziehungen mutete mit einem Mal als Relikt einer vergangenen Entwicklungsstufe der Ästhetik an. Damit war die einstmals bahnbrechende Innovation, die als ultimative Herausforderung an das verkrustete Literatursystem konzipiert worden war, als Verfahrensweise endgültig verfügbar geworden und in das Repertoire möglicher Ausdrucksregister eingegangen. ${ }^{\text {G4 }}$

Immerhin lebte die Gattung in ihrem epigonalen Sektor weiter. Hier wurden unermüdlich jene Gestaltungsmuster repetiert, welche die von der Avantgardeästhetik auf die Spitze - und in die Aporie - getriebene Transgressionslogik der Modeme

59 Stéphane Mallarmés Coup de dès (1897) stellt den Prototyp für die optisch umgesetzte Störung von Syntax und Leserichtung eines Textes dar. Mit diesem einzigartigen Experiment sind die "Selbstverständlichkeiten unserer Lese-, Schrift- und Buchkultur [...] in den Avantgarden reflexiv und infolgedessen als konstruktives Material verfügbar und semantisierbark geworden; Georg Jäger. Die Avantgarde als Ausdifferenzierung des bürgerlichen Literatursystems. Eine systemtheoretische Gegenüberstellung des bürgerlichen und avantgardistischen Literatursystems mit einer Wandlungshypothese. In: Michael Titzmann (Hrsg.): Modelle des literarischen Strukturwandels. Tübingen: Niemeyer 1991 (= Studien und Texte zur Sozialgeschichte der Literatur 33), S. 236. Aufgegriffen, variert und popularisiert wurde Mallarmés Neuerung dann von den Folgeavantgarden des frühen 20. Jahrhunderts.

60 Siehe hierzu die Ausführungen zum frühen 20. Jahrhundert bei Christina Weiss: Seh-Texte. Zur Erweiterung des Textbegriffes in konkreten und nach-konkreten visuellen Texten. Hrsg. vom Institut für Modeme Kunst, Nümberg. Zimdorf: Verlag für Modeme Kunst 1984.

61 Vgl. Christian Scholz: Untersuchungen zur Geschichte und Typologie der Lautpoesie. 3 Bde. Obermichelbach: Scholz 1989.

62 Kiefer deutet denn auch den "Surrealismus als Krise der Moderne«; Klaus H. Kiefer. Diskurswandel im Werk Carl Einsteins. Ein Beitrag zur Theorie und Geschichte der europäischen Avantgarde. Tübingen: Niemeyer 1994 (= Communicatio 7), S. 366.

63 Vgl. hierzu Hans Burkhard Schlichting. Pioniere des Medialen. Zur Aktualität der dadaistischen Kultur-Attacke. In: Kultur. Bestimmungen im 20. Jahrhundert. Hrsg. von Helmut Brackert und Fritz Wefelmeyer. Frankfurt a.M.: Suhrkamp 1990 (= edition suhrkamp N.F. 587), S. 32-85.

64 Vgl. hierzu auch Peter Bürger: Das Altern der Modeme. In: Adomo-Konferenz 1983. Hrsg. von Ludwig von Friedeburg und Jürgen Habermas. Frankfurt a.M.: Suhrkamp 1983 (= suhrkamp taschenbuch wissenschaft 460), S. 177-197. 
längst shinter sich gelassen hatte. Auf diese Weise funktionierte ausgerechnet die triviale Zirkulationssphäre des Genres als eine Art kulturelles Formengedächtnis, das einstmals etablierte Gestaltungsweisen aufspeicherte und über einen Zeitraum, in dem die Textsorte im Sektor »eingeschränkter Produktion« vom Verschwinden bedroht war, tradierte. Bevor es aber zu einer erneuten Revitalisierung des Gattungsmusters kommen konnte, kappte die von den Nationalsozialisten betriebene dezidiert antimodernistische Literaturpolitik auf brutale Weise den Bezug zu den möglichen eigenkulturellen Vorbildern wie auch den Austausch mit der Kunstentwicklung im Ausland und besiegelte so den tiefen Bruch in der Gattungsgeschichte, der sich bereits abgezeichnet hatte. ${ }^{65}$

Erst im Zuge einer Rekonstruktion der Moderne, ${ }^{66}$ wie sie sich nach Ende des Zweiten Weltkriegs in der Bundesrepublik und - obzwar verzögert und abgeschwächt - in der DDR gleichermaßen ereignete, kam es seit den fünfziger Jahren in der deutschen Literatur zu punktuellen Wiederaufnahmen des Gattungsmodells Prosagedicht. Bezogen auf die Bundesrepublik wären hier vor allem Autoren wie Günter Eich, Walter Helmut Fritz, Günter Bruno Fuchs und Hans-Jürgen Heise zu nennen, innerhalb der DDR-Literatur Schriftsteller wie Johannes Bobrowski, Helga M. Novak (1966 ausgereist), Ulrich Berkes und Sarah Kirsch (1977 ausgereist). Da sich trotz diverser Restaurationsversuche die Geltungskraft einer normativen Gattungsordnung in der Nachmoderne aber nicht mehr durchzusetzen vermochte, fehlt ein Faktor für eine wirklich breite Nutzung dieser Textsorte. Gleichwohl verschaffte die weiterhin bestehende dichotomische Spaltung des Literatursystems dem Prosagedicht zahlreiche Refunktionalisierungsoptionen. Als historisch gewordener Bestandteil des verfügbaren Kanons subversiver Überschreitungsgesten steht der Einsatz des zwischen sPoesie und Prosa, Lyrik und Nichtlyrik angesiedelten Genres seitdem im Zeichen zitierter Moderne. Doch das ist eine andere Geschichte.

65 Mit den Dichtungen in V'ers und Prosa (1838) von Hans Kurz liegt das späte Beispiel einer entsprechenden Mischpublikation eines deutschen Autors vor.

66 „Von einer nekonstruierten Moderne ist zu sprechen, da [...] davon auszugehen ist, daß die Modeme in der Literatur der unmittelbaren Nachkriegszeit verschüttet war oder gar, wie in der westdeutschen Nachkriegsgermanistik, absichtsvoll vermieden wurde. Zum Teil mußte sie unter sehr unterschiedlichen Bedingungen und Behinderungen von Autoren [...] nachgeholt und nachgearbeitet werden.« Klaus R. Scherpe: Die rekonstruierte Moderne. Studien zur deutschen Literatur nach 1945. Köln/Weimar/Wien 1992 (= Literatur - Kultur - Geschlecht, Bd. 3), S. XIV. Die Herausgeber der Festschrift für Hans Zeller konstatieren für die Zeit nach 1945 eine 'Zweite Modernes, in der - bis in die sechziger Jahre hinein - Literaturentwicklungen in der BRD im Zeichen der Entdeckung und Aneignung der sog. Klassiker der Moderne stünden; vgl. Aus dem Marbacher Magazin gehobene Daten \& Fakten, Reden, Verlautbarungen \& Veröffentlichungen zur Geschichte der Deutschen Schillergesellschaft, des Schiller-Nationalmuseums, des Deutschen Literaturarchivs \& der Sammlungen in den Jahren 1953 bis 1979; 19.9.1979 Bemhard Zeller überreicht von seinen Mitarbeitern. Stuttgart: o.V. 1979 (= Marbacher Magazin). 